\title{
Developing RFID-based Systems for Security in Marine Transportations
}

Kyprianos Papadimitriou $\dagger$, Dionisios Pnevmatikatos $\dagger$, Apostolos Dollas $\dagger$, Christos Koulamas $\ddagger$, Stavros Aggelidaki*, Petros Chatzidakis*, Leonidas Vasilikiotis $\diamond$, Maria Emmanouilidou $\diamond$

\author{
$\digamma$ Technical University of Crete \\ \{kpapadim, pnevmati,dollas\}@mhl.tuc.gr
}

$\ddagger$ Industrial Systems Institute

koulamas@isi.gr

\author{
*ANEK Lines \\ m.nomikos@anek.gr \\ $\diamond$ Hellenic Seaways \\ l.vasilikiotis@hsw.gr
}

\begin{abstract}
In marine transportations it is important to control effectively the boarding and debarkation of passengers and vehicles, as well as to effectively monitor the cargo. These procedures are usually handled using conventional ways such as barcode-based tickets and scanners. This technology, although used widely, has significant drawbacks and is outdated. In the present project we propose the use of Radio Frequency Identification Technology (RFID) technology to control these procedures. This paper describes the initial concept, the possible ways to fit the RFID technology in marine sector and the initial considerations on system architecture. We discuss the benefits of RFID over the barcode-based system in the context of the specific domain, the problems that may arise with its use and possible ways to tackle them. The research is carried out within the framework of the ongoing project "MERIT" 1 .
\end{abstract}

\section{INTRODUCTION}

Vessels are one of the dominant transportation means for cargo worldwide and for passengers in countries such as Greece. Two main procedures that need to be handled effectively are boarding and debarkation, similar to the check-in and check-out that take place in other types of transportation such as air carriers. However, in the marine sector due to inherent restrictions imposed by the large amount of load, i.e. passengers, vehicles and cargo, it is difficult to follow similar strategies. Hence, operations such as manual counting of the passengers, vehicles and luggage (the so called units) cannot apply during boarding and debarkation due to the amount of units that need to be served in limited time. In fact, boarding should be completed relatively shortly, while debarkation needs to be performed even faster. At the same time, it is desirable for the a maritime company to be aware of the amount of units entering and exiting the vessel. This entails accuracy in the check-in/out procedures. Also, it is necessary to maintain passengers' convenience and achieve high processing throughput. Another characteristic of the Greek maritime operation is that in many routes there are several stopovers (intermediate ports) where loading and unloading of passengers, vehicles and cargo (either small such as personal luggage or bigger items such as containers) are carried out. This burdens even more the task of controlling the vessel

\footnotetext{
${ }^{1}$ The financial support received through the General Secretariat for Research and Technology under Grant No. 09SYN-52-658 is gratefully acknowledged.
}

condition with respect to the awareness of the exact load at any point of time.

Today, check-in is performed using conventional barcode technology. Indicatively, a passenger enters the vessel and an employee scans his/her barcode ticket to check its validation. A similar process is applied for loading the vehicles and cargo into the vessel. It is obvious that the use of manual means for controlling boarding and debarkation is a timeconsuming process, while it lacks security when it comes to check the matching of passengers' ID with the personal information printed on the ticket. In addition, it is very difficult or even impossible to count accurately the amount of passengers getting out/in the vessel in intermediate port stations as currently debarkation is not connected at all with the counting and identification of the units exiting the vessel. In particular, the check-out procedure is missing completely.

Present work aims at designing a system relying on RFID technology to leverage the control of boarding and debarkation. A prototype will be developed to secure the check-in/out procedure, while it will enable the awareness of the vessel load at any point of time. In addition, if the new technology will enable a less painful procedure for the passengers and the employees as compared to the barcode technology, it could displace the latter completely. The contributions of present paper are:

- the use of RFID technology in controlling the checkin/out procedures in vessels,

- initial solutions to adjust RFID technology in the maritime domain, while at the same time avoiding radical changes in the vessel morphology and the existing IT infrastructure,

- $\quad$ the system architecture in an abstract level.

The paper is structured as follows: Section II describes the boarding and debarkation procedures currently followed by the maritime companies. Section III is devoted to the main scope of the work and the initial concept of the project and identifies the limitations to be considered. Section IV presents the proposed approach, while Section V concludes the paper and points out the next steps of the project. 


\section{PRESENT StATUS IN BoARding AND DEBARKATION}

This Section discusses the operational procedures followed during boarding and debarkation along with their problems and limitations. Then it identifies the problems that can be solved with the use of RFID technology, while it points out the ones requiring a more delicate solution.

A ticket is equipped with barcode technology which encodes the personal information of the passenger along with some details for the trip. Such information is a unique-per-trip number, the name and age of the passenger, the departure date, vessel name, arrival and destination port. This information is also printed on the ticket. While a person enters the vessel, an employee scans the ticket with the barcode scanner. The result is sent to the Central Data Base (CDB) of the maritime company (through a wireless connection such as WiMax, 3G or Satellite) so as to verify the ticket based on the data stored in the Central Data Base. These data were stored in the CDB at the time the ticket was issued. Once there is a match, the ticket is confirmed. At the same time the person should be identified using a valid personal document, e.g. ID or passport. In specific it is checked whether the information printed on the ticket matches the ID of the person. Then the person is allowed to board the vessel and is considered as a passenger. It should be noted that in case the ticket is not valid, or the printed information does not match the ID, the person is instructed to leave the vessel, i.e. is not confirmed as passenger. From the aforementioned description, it is obtained that during boarding there exists personnel holding specific positions in the vessel for supervising and controlling the procedure. Thus the procedure is completely manual.

Normally, someone buys a ticket from a booking office, and arrives at the port at least one hour ahead of the departure. There are cases in which a person or a vehicle needs to be boarded just a few minutes before the departure. Maritime companies have expressed their will to continue offering this "last-minute" option. Also, this is a rule imposed by the maritime regulations and holds for all units with valid tickets.

During debarkation the passengers exit the vessel without following a certain procedure. Thus check-out or even counting of the passengers is not performed. Instead, personnel are assigned to search the entire vessel for remaining passengers.

With regard to the stopovers, there are passengers walking out of the vessel that should return prior to the scheduled departure time, e.g. passengers willing to shortly visit the place. However, there are cases in which passengers haven't returned prior the departure time. As mentioned previously, the procedure lacks check-out mechanism, so there is no way to be aware of the amount and the identity of the passengers that haven't returned. Consequently, it is very likely for the vessel to depart without them.

Therefore, changes are needed to handle boarding and debarkation in an effective and secure manner. The checkin/out procedure followed in other sectors, e.g. air carriers, does not fit well in the maritime sector. Especially head counting cannot be considered as an option (it is controllable in an airplane due to its size) when it comes to serve vessels with a load of over 1800 people and 600 vehicles (data provided by ANEK Lines and Hellenic Seaways) plus a considerable amount of cargo.

With regard to the cargo the following procedure is applied. A person buys a barcode tag from the maritime company's kiosk at the port outside the vessel; then, this tag is adhered on the luggage. The encoded information contains a uniqueper-trip number, details of the trip (date, vessel name, arrival and destination port) and the name of the recipient. In the case of unaccompanied luggage, the name of the sender and the recipient is encoded and printed on the sticker. Then the person gets the luggage to the storage area, usually located on the ground floor of the vessel. An employee scans the barcode sticker and stores the luggage. The exact same process is followed either if the luggage belongs to the passenger or it is unaccompanied. The only difference is that in the former case the name of the sender and the recipient is the same, while in the latter case it isn't. In the second case the person is essentially a client and gets a receipt imprinted with the name of the recipient. Once the vessel arrives at the destination port, the recipient picks up the luggage after being identified using an ID.

A similar process is followed in vehicle boarding. A barcode ticket with a unique number should have been issued, imprinted with information of the vehicle such as its registration number and brand. In order to allow a driver boarding a vehicle into the vessel, an employee standing at the garage entrance checks if the vehicle matches with the information printed in the ticket. It should be noted that vehicles are not allowed to enter the vessel in the case of late arrival, so "last-minute" policy is not applied here due to potential congestion in arranging the vehicles. Check-out process for the vehicles is missing as well.

It turns out that boarding and debarkation are currently controlled in a completely manual way. For each unit a barcode ticket is issued. We aim at automating part of the procedure, so as to make it less painful for the personnel and the passengers. At the same time, we want to increase the security level with regard to the awareness of the load, e.g. knowing the exact amount of passengers debarked in an intermediate station that should return before the vessel's departure. Changes are needed to overcome limitations coming from the use of barcode technology. To recap, the following stimulate mainly our work:

- Manual check-in can be error-prone as it relies completely on the human factor. Also it causes delays in boarding.

- Problems are caused when a person is not allowed to pass and leaves the vessel. As reported by the maritime companies, this can cause confusion and delays in servicing the passengers' line. 
- Check-out procedure is completely missing and manual counting is not an option. This is a major problem, e.g. some passengers can hide in the vessel to cause damages, and then leave.

- Passengers exiting the vessel in intermediate ports are not identified or counted. This inhibits the awareness of the amount and identity of the passengers that should return before the scheduled departure. This is a consequence of lacking a check-out mechanism.

- Luggages in the storage area are not guarded.

We expect that some of the above problems will be solved completely with the incorporation of RFID technology. For example, it will eliminate the manual scanning of barcode tickets, while identification and counting of RFID tags is much more accurate as it doesn't rely on the human factor. By controlling the procedure with automatic RFID means during the boarding and debarkation at the points of entrance and exit respectively, we expect to enable the awareness of the load of the vessel at any point of time. This will be feasible with the introduction of check-out process in debarkation.

\section{SCOPE OF WORK}

Focusing on the applications of RFID tickets, as they have been implemented to date, the main motivation is to automate the embarkation process and reduce stowaways. Second, technically, implementation is done using contactless smart cards or NFC (Near Field Communication) cards, which requires the card to be in less than $10 \mathrm{~cm}$ from the reader to be recognized. The latter may address particular requirements of the embarkation phase (e.g. opening a door in a corridor) but does not allow the exploitation of other features of relevant technologies, as the far field communication capabilities of UHF band RFID which may be applied in order to also track a more massive people debarkation procedure.

This work aims to present an integrated solution based on RFID technology, exploiting the maximum characteristics and covering such simultaneous, multiple readings during the debarkation, introducing auto-identification in combination with the foreseen availability of electronic identity cards/passports (e-passports), and studying it in all of its dimensions, economic, technical and social, according to the current European Commission guidelines [1-4]. One of the main overall objectives, apart from speeding up and improving the current procedures, is to increase the safety level in maritime transportations by keeping an accurate record of the number and identities of all passengers in the vessel, a not so straightforward and even existing today procedure considering the large number of intermediate stops in Greek maritime routes.

Some of the critical issues that arise and need to be effectively addressed are:
- How to achieve a correct identification based on the listed names on the tickets, especially without the wide availability of e-passports today?

- What are the requirements for real-time connectivity between the on-vessel IT equipment and the central enterprise data bases, and what happens when this connectivity is disturbed?

- How to apply the restriction that every passenger should carry his/her ticket or boarding pass during debarkation (something completely different from the present situation)?

- What are the procedures covering a ticket loss?

- Which are the most important added value services that could be linked with such an electronic ticket and how to exploit them in order either to improve the quality of services or to solve other issues (e.g. convince people to keep tickets while exiting the vessel)?

- Which are the implications from storing personal data when and where this is needed, and how to safeguard them while being in conformance with the regulatory framework?

- Which are the similarities and differences in applying relevant procedures in tracking also vehicles, packages, containers, etc., via the same technology?

\section{A. Security and privacy}

Unauthorized access to personal information potentially contained in the ticket or connected with it, or monitoring the position and behavior of RFID ticket holders, and organization and combination of large volumes are some of the main threats that concern people today. Addressing these threats and ensuring the necessary privacy conditions must be done at three levels: the legal level, the business process level and the technical level [4]. Regarding the technical level, since there is a wireless transmission of an object identifier and not only of its class, and as the typical tag's resources are very limited, the provision of guarantees over security and privacy in an RFID-based system are still quite challenging and must be investigated throughout the whole system structure. While there is an already active research and even existing product features in implementing key processes of encryption and validation of access rights at the level of RFID communications, the requirement to use RFID technology tags with widely available features and with an acceptable cost lead to an exploration of solutions targeting the remaining parts of the architecture, from the RFID reader and up [5-8].

The MERIT consortium focuses on the problem of ensuring the conditions of privacy, addressing it directly to two of the three levels of immediate access, that is, on the technical and on the internal business process levels, while it seeks to also 
affect the legal level, by recording all potential problems that can be solved optimally with the assistance of a legislative framework. In terms of procedures, the project contribution is an original application for the use of RFID technology in shipping and the safe embarkation/debarkation, including stopovers. Emphasis will be placed firstly on the boarding procedures and on the ticket issuance ones, including the consequences on a large-scale future implementation beyond the pilot case and how these may affect the complex network of ticketing agencies. In technical terms, this is translated into specific requirements, protocols and control mechanisms to be able to support business processes, leading to an electronic and information system which will ensure the privacy conditions by design. In particular, emphasis will be given to the overall architecture and to the middleware, including but not limited to address important open issues, as they are reported in the literature and in the current versions of specifications and international standards [9], including:

- The methods of access control and authentication between users, between individual agencies, between subsystems of the architecture and between readers and other equipment and devices

- The usage methods of basic mechanisms, such as the 'kill' and 'lock' command on labels and the subsequent management of passwords

- Methods for increasing the security of low-cost passive tags through the management of key aspects of the operation of the reader (e.g. power output) and filters (e.g. direct identification of non-authentic copies).

\section{B. RFID and wireless channel}

At the physical and hardware level, the various technologies of RFID tags are distinguished by the way they are powered and the way they communicate with the readers (passive/active), the level of functionality and service (read/write, memory, processing capabilities, etc.) and the operating frequency and protocols on the wireless medium. The obvious cost increase, when the capabilities of the tags / cards increase, too, provides a first level of trade-offs against the required functionality that need to be adopted for each application and scenario separately. In this study, however, a not-so-obvious level of performance evaluation of communication processes between tags and readers [10] must be included, too, which extends from the necessary comparison between technologies and standards to a comparison also between different implementations [11].

In the direction of the study and performance improvements of RFID communications, major research efforts exist [1221]. The reported factors that affect the final behavior and performance of RFID communications include the nature of materials and the layout of the environment, the number, topology installation and the adjustment of readers, but also the selection rules and the various implementations of the antennas in the readers and in the cards. Taking also into account mainly the heavily metallic construction of areas in a vessel, as well as the possible coexistence of other sources of wireless transmissions, either in the same system or in applications in adjacent vessels or in the port, the MERIT consortium addresses research on performance measurements and analysis on the system behavior in the inherently unfriendly to wireless communications environment of a vessel.

\section{PROPOSED APPROACH}

In order to justify the use of RFID technology in maritime operations we need to solve problems related with the security and the delays incurred by the barcode technology, and to provide additional features such as an effective checkout procedure. We also need to examine the limitations coming from both the side of maritime companies and ultimately from the side of passengers. With regard to the formers we do our best to respect their operations and infrastructure and avoid making major changes. With regard to the passengers we aim to avoid increasing their frustration during boarding and debarkation, and to offer extra services supported by RFID technology.

The companies are concerned with the changes needed in their operations to adopt the RFID technology. They want (i) to continue offering the "last-minute" boarding, (ii) they do not want to modify their IT infrastructure, (iii) they prefer to avoid changing the internal morphology and structure of the vessel, and (iv) they don't want to supply the booking offices and third-party travel agencies with RFID special equipment.

To tackle the latter issue (iv), we decided to introduce the notion of check-in/check-out by using RFID boarding passes. In particular, to avoid changing completely the process we will leave intact the process of ticket issuance in the ticket offices/agencies. On the other hand, a person will not be able to use this ticket for boarding. Instead, in order to be eligible to travel he/she should obtain an RFID boarding pass by a kiosk located close to the vessel by showing a valid ticket and ID. Hereinafter, terms such as RFID boarding pass, RFID ticket and RFID tag will be used interchangeably.

With regard to the internal structure of the vessel (iii), adjustments are needed to accommodate boarding and debarkation. We consider the use of two RFID subsystems in two different spots close to the gate (called RFID gates), instead of one. Thus only in the case a person will pass through both the RFID gates will be confirmed as a passenger. This is illustrated in Figure 1 and solves the problem described in the following scenario: Suppose that only one RFID gate is used. If a person with a valid RFID boarding pass approaches the RFID gate, but eventually turns back and leaves the vessel, the system will recognize him/her as a valid passenger. This problem is solved with the $2^{\text {nd }}$ RFID gate which is used to confirm the boarding, while the $1^{\text {st }}$ RFID gate annotates a person as an unconfirmed (potential) passenger. The swing gate placed in front of the $1^{\text {st }}$ RFID gate can be optionally used to control 


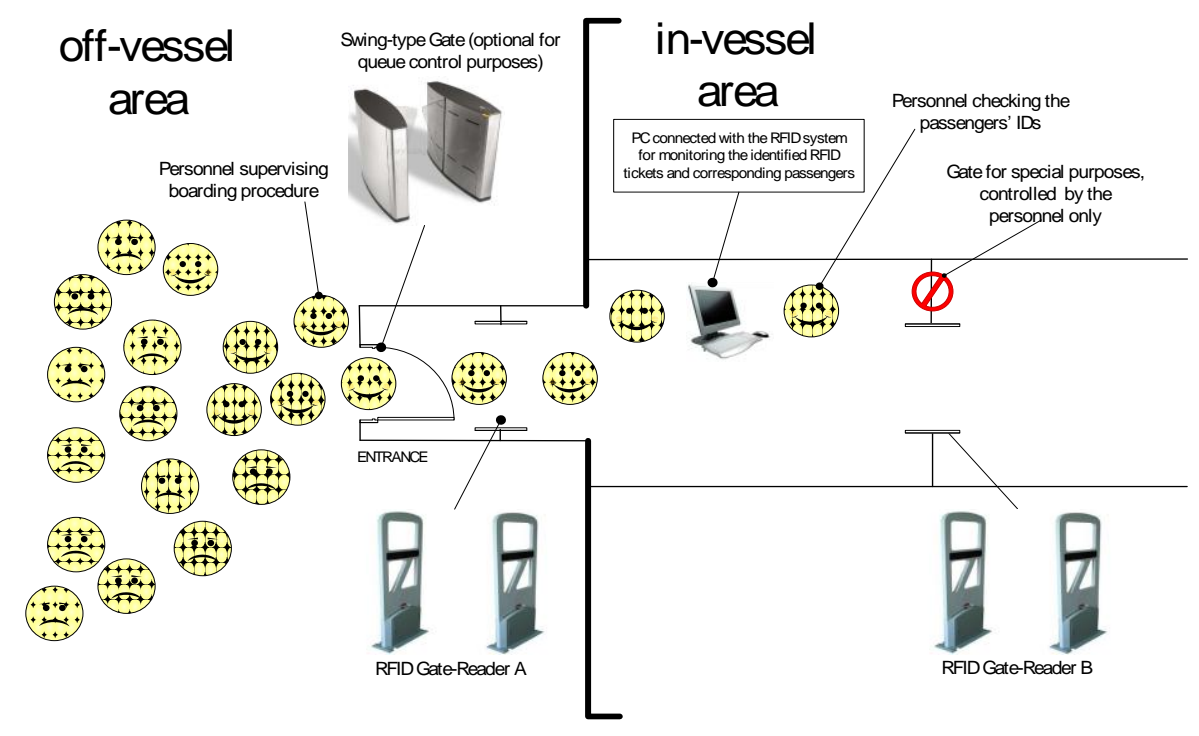

Figure 1: When a person passes through RFID Gate A is considered as unconfirmed passenger (within the controlled area). Only once the person passes the RFID Gate B is considered as confirmed passenger.

the rate of the passengers entering the controlled area, i.e. the area between the two RFID gates. The structure of Figure 1 provides an initial solution, however it needs to be studied from the aspect of cost. Towards this direction, we consider mobile solutions such as specialized RFID gates that can be easily hooked on/unhooked by the vessel during boarding and debarkation respectively.

The notion of confirmed and unconfirmed boarding and passengers has been introduced. A passenger is supposed to be in an unconfirmed status while being in between the two RFID gates, whereas after passing the $2^{\text {nd }}$ RFID gate he/she is considered to be in confirmed boarding status. The terms RFID interrogators, RFID readers and RFID gates, have the same meaning and are used interchangeably.

The existing IT infrastructure (ii) won't be affected at all. Communication between the local on-vessel RFID system and the CDB will be done using the existing interface. The only change concerns the replacement of barcode scanner by the RFID interrogator, while the latter's interface will be adjusted to the current infrastructure.

From the passengers' side, we are seeking ways to reinforce the smooth acceptance of the RFID boarding pass. We deem necessary to provide motivations to the passengers in adopting this technology. For example, a way to convince the passengers to carry the RFID tickets when exiting the vessel at intermediate port stations, is to inform them about the exact functionality in the check-out procedure. Essentially, someone holding an RFID ticket will be tracked in a specific point only, i.e. the exit/entrance of the vessel. Hence, the system will be aware of which passenger has or hasn't returned from his/her looking-around walk at the intermediate port, prior to the scheduled departure time. Towards the same direction we consider added-value services that can be offered with RFID technology. Fine candidates could be high-level services such as targeted marketing according to the passengers' profile. For example, once a specific RFID is detected close to shopping spots, an SMS can be sent to the passenger's cell phone informing him/her about discounts on a brand of clothes matching the passenger's preferences, special offerings in books and so on. As targeted marketing relies on the profile of the passenger, this profile needs to be stored in the CDB per passenger's consent.

Some problems are raised due to the automatic way the RFID system tracks the passengers holding RFID tickets. A complex case can occur when one passenger carries more than one valid RFID tickets (for the same trip), e.g. a parent who holds the RFID tickets of all the family members. This needs a further study and it is likely that similar situations will occur within the controlled area (in between the two RFID gates of Figure 1). A potential delay in the passengers' line might be incurred in this case, and it is worthwhile to analyze such extreme cases and identify the worst ones.

Present work is involved with numerous tasks ranging from the assessment of different RFID hardware, the software to support it properly, the middleware that should communicate with the existing CDB in a seamless way, and examination of the potential interference of RFID components with the metallic body of the vessel and other wireless sources (hence, it is necessary to choose the equipment that suits our case). In addition, privacy and security constitute high-priority tasks in our project due to the critical data stored in the memory of the RFID tickets which might be exposed to attacks aiming at copying them.

\section{CONCLUSIONS AND ONGOING WORK}

In this paper we give an overview of the issues in boarding and debarkation of passengers, vehicles and cargo in vessels 
focusing on the peculiarities of the Greek shipping environment. We also outlined the approach investigated in the "MERIT" project, which is in its early exploration stage. We intend to build a complete testbed system and deploy it on two vessels, one of ANEK and another of HSW. This pilot system will allow us to collect actual measurements and focus not only on the technical aspects of RFID technology and equipment, but also on system-wide effects such as customer satisfaction, boarding/debarkation process speed and efficiency, and necessary personnel.

\section{REFERENCES}

[1] Comission of the European Communities, Brussels, 12.5.2009, 3200 final Commission Recommendation of 12.5.2009 on the implementation of privacy and data protection principles in applications supported by radio-frequency identification

[2] RFID Research Needs, Cluster of European RFID Projects, CERP, http://www.rfid-in-action.eu/cerp, June 2007

[3] Florent Frederix, DG INFSO, European Commission, "Theme: Internet Evolution and Security. Strengthening SME competitive advantage through RFID implementation", 2009

[4] Marc van Lieshout et al, "RFID Technologies: Emerging Issues, Challenges and Policy Options", JRC Scientific \& Technical Reports, European Commission JRC, IPTS, EUR 22770 EN, 2007

[5] Juels, Ari Weis, Stephen A, "Defining Strong Privacy for RFID", Fifth Annual IEEE International Conference on Pervasive Computing and Communications, PerCom Workshops, March 2007, pp. 342-347

[6] Sepideh Fouladgar, Hossam Afifi, "Scalable privacy protecting scheme through distributed RFID tag identification", Proceedings of the workshop on Applications of private and anonymous communications, Instabul, Turkey, 2008

[7] J. Aragones-Vilella, A. Martinez-Ballest and A. Solanas, "A Brief Survey on RFID Privacy and Security", Proceedings of the World Congress on Engineering, WCE 2007 Vol II, July 2007, London, U.K.

[8] Juels, A., "RFID security and privacy: a research survey", IEEE Journal on Selected Areas in Communications, Vol.24, Is.2., Feb. 2006, pp: 381- 394

[9] The EPCglobal Architecture Framework, EPCglobal Final Version 1.3, March 2009, http://www.epcglobalinc.org

[10] "Common RFID Implementation Issues: 10 Considerations for Deployment", white paper, www.alientechnology.com

[11] Zoch, W. and Ferguson, D. "RFID Performance Testing, Beyond Compliance. The Importance of Pre-Deployment, Customer-Specific Performance and Integration Testing to RFID", White Paper, Sensormatic, www.sensormatic.com, August 2004.

[12] Ricardo Meneses, Laura Montes and Roberto Linares, "The RFID Radio Channel Performance in the Vehicular Control", Journal of Vectorial Relativity, JVR 4, 3 78-38, September 2009.

[13] Sohraby, K., Daneshmand, M., Chonggang Wang, Bo Li, "Performance analysis of RFID Generation-2 protocol", IEEE Transactions on Wireless Communications, Vol 8, Issue 5, pp. 2592-2601, May 2009.

[14] Chonggang Wang, Mahmoud Daneshmand, Kazem Sohraby, "Optimization of tag reading performance in generation-2 RFID protocol", Computer Communications, Volume 32, Issue 11, pp. 1346 1352 , July 2009.
[15] Jeong Geun Kim, Woo Jin Shin and Ji Ho Yoo, "Performance Analysis of EPC Class-1 Generation-2 RFID Anti-collision Protocol", Lecture Notes in Computer Science, Computational Science and Its Applications - ICCSA 2007, Springer Berlin / Heidelberg, Volume 4707/2007, pp. 1017-1026, August 29, 2007.

[16] Pavel V. Nikitin, K. V. Seshagiri Rao, Rene Martinez, and Sander F. Lam, "Sensitivity and Impedance Measurements of UHF RFID Chips", IEEE Transactions on Microwave Theory and Techniques, Vol. 57, No. 5, May 2009

[17] Michael Buettner, David Wetherall, "An Empirical Study of UHF RFID Performance”, MobiCom'08, September 14-19, 2008, San Francisco, California, USA.

[18] You-Chang Ko, Sumit Roy, Joshua R. Smith, Hyong-Woo Lee, and Choong-Ho Cho, "RFID MAC Performance Evaluation Based on ISO/IEC 18000-6 Type C", IEEE Communications Letters, Vol. 12, No. 6, June 2008.

[19] Steve Hodges, Alan Thorne, Hugo Mallinson, and Christian Floerkemeier, "Assessing and Optimizing the Range of UHF RFID to Enable Real-World Pervasive Computing Applications", Pervasive 2007, LNCS 4480, pp. 280 - 297, Springer-Verlag Berlin Heidelberg 2007.

[20] Iain A. Currie, Mahesh K. Marina, "Experimental evaluation of read performance for RFID-based mobile sensor data gathering applications", Proceedings of the 7th International Conference on Mobile and Ubiquitous Multimedia, Pages 92-95, Sweden, 2008.

[21] Clark Robert H., Twede Diana, Taxelaar Jeffrey R., Boyer Kenneth K, "Radio frequency identification (RFID) performance : The effect of tag orientation and package contents", Packaging technology \& science journal, ISSN 0894-3214, 2006, vol. 19, no1, pp. 45-54. 